\title{
Lattice Boltzmann and Jones matrix calculations for the determination of the director field structure in self-propelling nematic droplets
}

\author{
Christian Bahr $\odot^{*}$ \\ Max Planck Institute for Dynamics and Self-Organization, Am Faßberg 17, D-37077 Göttingen, Germany
}

(Received 28 July 2021; accepted 24 September 2021; published 13 October 2021)

\begin{abstract}
Nematic droplets immersed in aqueous surfactant solutions can show a self-propelled motion induced by a Marangoni flow in the droplet surface. In addition to the self-propulsion, the Marangoni flow induces within the droplet a convective flow which considerably influences the nematic director field of the droplet. We report numerical simulations aiming at the determination of the director field in the self-propelling droplet. The convective flow and the resulting structure of director field are described by a lattice Boltzmann model. The reliability of the obtained structures is proved by subsequent Jones matrix calculations which enable the direct comparison of experimental polarizing microscopy images of self-propelling droplets with calculated images based on the simulated structures.
\end{abstract}

DOI: 10.1103/PhysRevE.104.044703

\section{INTRODUCTION}

Artificial microswimmers based on liquid droplets selfpropelling in a second liquid have gained attention as model systems for the study of biological microswimmers ranging from the single swimmer level to the collective behavior [1]. A versatile class of droplet swimmers consists of cyanobiphenyl compounds: small rod-shaped organic molecules which are well known as thermotropic liquid crystals. Nematic liquid crystals are liquid phases in which the rod-shaped molecules tend to align along a common direction, specified by a unit vector $\vec{n}$ named director. A standard compound is 5CB (4-pentyl-4'-cyanobiphenyl), which is used in numerous fundamental and application-oriented studies because it exhibits the nematic phase at room temperature.

Recently it was observed that cyanobiphenyl compounds can serve, beyond their liquid crystal properties, as artificial microswimmers: If a 5CB droplet (typical diameter $50 \mu \mathrm{m}$ ) is placed into an aqueous solution of standard ionic surfactants such as hexadecyltrimethylammonium bromide (CTAB) or sodium dodecyl sulfate (SDS), a spontaneous solubilization process, consisting of the transfer of 5CB molecules from the parental droplet into surfactant micelles in the aqueous environment, sets in provided the surfactant concentration is above a certain level (several times the critical micelle concentration) [2]. The micellar solubilization of the droplet is accompanied by a self-propelled motion which is induced by a Marangoni stress caused by a self-sustained gradient in the

\footnotetext{
*christian.bahr@ds.mpg.de
}

Published by the American Physical Society under the terms of the Creative Commons Attribution 4.0 International license. Further distribution of this work must maintain attribution to the author(s) and the published article's title, journal citation, and DOI. Open access publication funded by the Max Planck Society. surfactant coverage of the droplet surface [3]. In addition to the self-propulsion, the Marangoni flow in the droplet surface leads to a convective flow within the moving droplet.

The droplet swimmers described above possess several properties which render them especially versatile model systems for biological microswimmers. For instance, they show chemotaxis $[4,5]$ and exhibit collective behavior by forming floating clusters [6], and their surface flow pattern, induced by the Marangoni stress, can be changed from a simple neutral squirmer type (dipolar symmetry) to higher modes of, e.g., quadrupolar symmetry [7].

The compound $5 \mathrm{CB}$ is at room temperature in the nematic liquid crystal state and transforms into the isotropic liquid phase when the temperature rises above $35^{\circ} \mathrm{C}$. The self-propulsion and the above described features are present regardless of the phase of the swimming droplet, but the presence of nematic ordering influences considerably the swimming behavior: Whereas the motion of isotropic droplets is characterized by ballistic on short and diffusive behavior on long scales, nematic droplets move on helical or curling trajectories because of an asymmetry in the nematic director field [8]. The ballistic or diffusive behavior is recovered when the droplet diameter decreases [9]. Also, the presence of nematic order makes the droplet swimmers susceptible to external magnetic fields [10], and it enables the design of self-propelling shells [11].

The striking effects of the nematic order on the swimming behavior of the self-propelling droplets or shells result from the mutual coupling between flow and nematic order. While their swimming behavior is qualitatively well understood [8,11], a complete quantitative understanding requires the knowledge of both the nematic director field and the flow velocity field in the droplet. Experimentally, some information on the structure of nematic droplets, such as the type and position of topological defects, can be obtained from polarizing microscopy and the flow field could be accessible through PIV (particle image velocimetry) measurements. On the theory 
side, studies of droplet swimmers are usually concerned with isotropic droplets [12-14]. Recently, Mozorov and Michelin [15] have presented a minimal model which addresses the special features of nematic droplet swimmers. Here we propose an approach based on numerical simulations in order to obtain detailed information on the structure and the flow field in self-propelling nematic doplets: the lattice Boltzmann (LB) method is used to compute flow and director fields and subsequent Jones matrix calculations enable a comparison with experimental results. In the present paper, we describe the general approach and focus mainly on the determination of the director field. The back coupling on the flow field and the resulting effects on the swimming behavior are left for a future study. The paper is organized as follows: In the next section, we consider the simulation of the convective flow in the droplet. In Sec. III we add the nematic order to the droplet and study the influence of the convective flow on the director field. Section IV describes Jones matrix calculations which confirm the reliability of the structures obtained from the LB simulations. The last section provides a discussion and conclusion.

\section{FLOW IN SELF-PROPELLING DROPLETS}

The flow in the self-propelling droplet is induced by the Marangoni flow in the droplet surface which is caused by a gradient in the surfactant coverage. Assuming an axisymmetric gradient, the simplest pattern of the surface flow is that of a neutral squirmer. A squirmer is a model swimmer which has been introduced to describe the motion of (nearly) spherical biological microswimmers such as volvox algae $[16,17]$. Whereas the bioswimmers move by the generation of metachronal surface waves, model squirmers propel by a prescribed surface velocity. For a neutral squirmer, the tangential velocity $v_{\theta}$ at position $\vec{r}$ in the surface is given by

$$
v_{\theta}=B_{1} \sin \theta
$$

where $\theta$ is the polar angle between $\vec{r}$ and the $z$ axis (which we take as the symmetry axis) and $B_{1}$ is a constant coefficient.

As a first approach to model the velocity field inside the droplet, we use the standard lattice Boltzmann (LB) method for simple Newtonian fluids [18,19]. The LB method is based on a rectangular lattice, to each lattice node a set of distribution functions $f_{i}$ is assigned which describe the density of fluid particles traveling with velocity $\vec{c}_{i}$ to a neighboring grid node. The density $\rho$ and velocity $\vec{v}$ of the fluid at each node are thus given by

$$
\begin{aligned}
& \rho=\sum_{i} f_{i}, \\
& \vec{v}=\frac{1}{\rho} \sum_{i} f_{i} \vec{c}_{i} .
\end{aligned}
$$

We use the D3Q15 set, i.e., for each node in our threedimensional grid 14 neighboring nodes are considered, $i$ takes values from 0 to 14 , and the velocity vectors $\vec{c}_{i}$ are $(0,0,0),( \pm 1,0,0),(0, \pm 1,0),(0,0, \pm 1),( \pm 1, \pm 1, \pm 1)$ in units of $\Delta x / \Delta t$ (lattice spacing per time step). The evolution of the distribution functions is obtained via a collision and streaming step:

$$
f_{i}\left(\vec{r}+\vec{c}_{i} \Delta t, t+\Delta t\right)=f_{i}(\vec{r}, t)+\Omega_{i}(\vec{r}, t) .
$$

The collision operator $\Omega_{i}$ describes a relaxation towards an equilibrium distribution $f_{i}^{\text {eq }}$ at rate $\tau_{f}$ :

$$
\Omega_{i}=-\frac{\Delta t}{\tau_{f}}\left(f_{i}-f_{i}^{\mathrm{eq}}\right)
$$

The equilibrium distribution functions $f_{i}^{\text {eq }}$ can be obtained as expansions in the velocity under certain constraints. It can be shown [18,19] that

$$
f_{i}^{\mathrm{eq}}=w_{i} \rho\left[1+3\left(\vec{v} \cdot \vec{c}_{i}\right)+\frac{9\left(\vec{v} \cdot \vec{c}_{i}\right)^{2}}{2}-\frac{3(\vec{v} \cdot \vec{v})}{2}\right] .
$$

The weighting factors $w_{i}$ are $\frac{2}{9}$ for $i=0, \frac{1}{9}$ for $i=1$ to 6 , and $\frac{1}{72}$ for $i=7$ to 14 .

An explanation of the boundary conditions is in order. A standard boundary condition in a LB simulation is the bounce back boundary corresponding to a solid wall with no-slip condition: a fluid particle, which meets the boundary with velocity $\vec{c}_{i}$, travels back with reversed velocity $-\vec{c}_{i}$ to the grid node from which it has started. In order to enable the particle to reach again its initial node within one time step, the boundary has to be located exactly halfway between two neighboring nodes, implying directly that the boundary has to be parallel to one of the grid axes. This condition cannot be fulfilled for a curved boundary and the implementation of an interpolation procedure is necessary. We use the method described by Bouzidi et al. [20] in order to establish a spherical boundary around our sample volume.

A flow in a LB fluid can be induced in several ways, e.g., a body force can be introduced [21] or a velocity can be assigned to a no-slip boundary [22]. In order to generate the convective flow in our model droplet, we use the latter method and assign a tangential velocity field according to Eq. (1) to the spherical boundary of our sample volume. The resulting flow field (Fig. 1) has the expected shape of an axisymmetric convection ring. There are two stagnation points at the surface, one at the leading pole $(\theta=\pi$ for the example shown in Fig. 1) and the second at the opposite pole at the rear of the self-propelling droplet.

For a neutral squirmer the internal (and external) flow field can be described analytically: it corresponds to a liquid sphere which moves, e.g., by sedimentation, at low Reynolds numbers within a surrounding liquid and has been treated decades ago [23]. The velocity components $v_{r}$ and $v_{\theta}$ of the internal flow field are given (in the laboratory frame) as

$$
\begin{aligned}
& v_{r}=V \cos \theta\left[\frac{2+3 \mu}{2(1+\mu)}-\frac{\mu}{2(1+\mu)} \frac{r^{2}}{R^{2}}\right], \\
& v_{\theta}=-V \sin \theta\left[\frac{2+3 \mu}{2(1+\mu)}-\frac{\mu}{(1+\mu)} \frac{r^{2}}{R^{2}}\right] .
\end{aligned}
$$

Here $V$ is the velocity of the sphere, $R$ is its radius, and $\mu$ is the viscosity ratio between inner and outer liquid. In order to obtain the flow field in the frame of the moving droplet, we would have to subtract the droplet velocity $v_{r}=V \cos \theta, v_{\theta}=$ $-V \sin \theta$. We can now make a direct comparison between the 


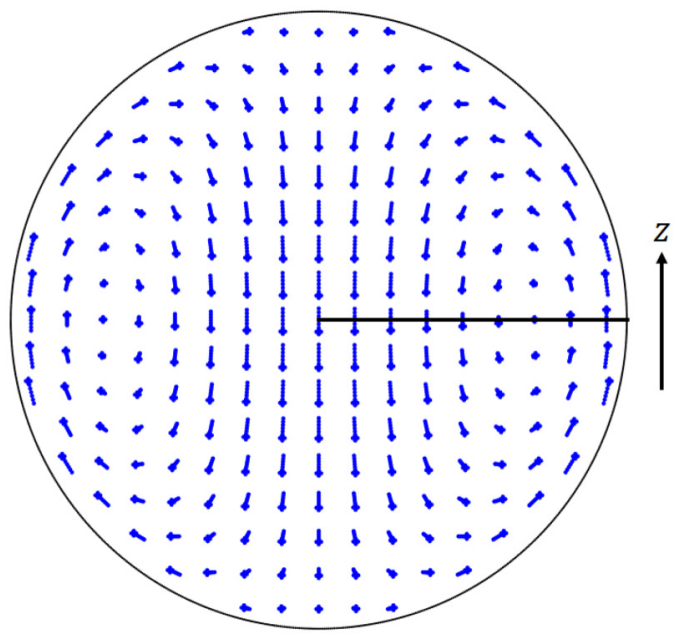

FIG. 1. Velocity field in a spherical sample volume resulting from the LB simulation with a surface velocity pattern according to Eq. (1). Blue arrows show the local flow velocity. Shown is an example with $B_{1}<0$, the droplet would move along the $-z$ direction. The black solid line indicate the locations of the data shown in Fig. 2.

results of the LB simulation and the analytical description. As shown in Fig. 2, there is a quantitative agreement.

Squirmers can exhibit higher modes in their surface velocity patterns $[17,24]$ which we can assign to our model droplet. Adding the second mode to Eq. (1) results in

$$
v_{\theta}=B_{1} \sin \theta+\frac{B_{2}}{2} \sin (2 \theta) \text {. }
$$

The ratio of the two coefficients, $B_{2} / B_{1}$, determines whether the squirmer shows the characterics of a "pusher" $\left(B_{2} / B_{1}<0\right.$, propulsion mainly from the rear) or a "puller" $\left(B_{2} / B_{1}>0\right.$, propulsion mainly from the front). The internal flow fields, resulting from the LB simulation, are shown in Fig. 3 (upper and middle panels). We can also generate asymmetric flow fields, e.g., by assigning a dependence on the azimuthal angle $\phi$ to the coefficient $B_{1}$ in Eq. (1). A possible equation for the

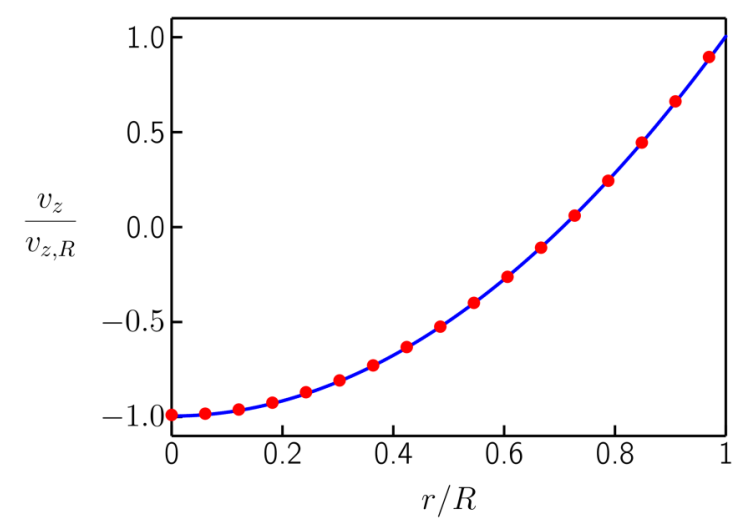

FIG. 2. Velocity component $v_{z}$ (in units of the surface velocity $v_{z, R}$ ) as function of distance $r$ (in units of droplet radius $R$ ) from the droplet center along the black line in Fig. 1. Red dots: data from LB simulation; blue line: analytical solution according to Eqs. (7) and (8).
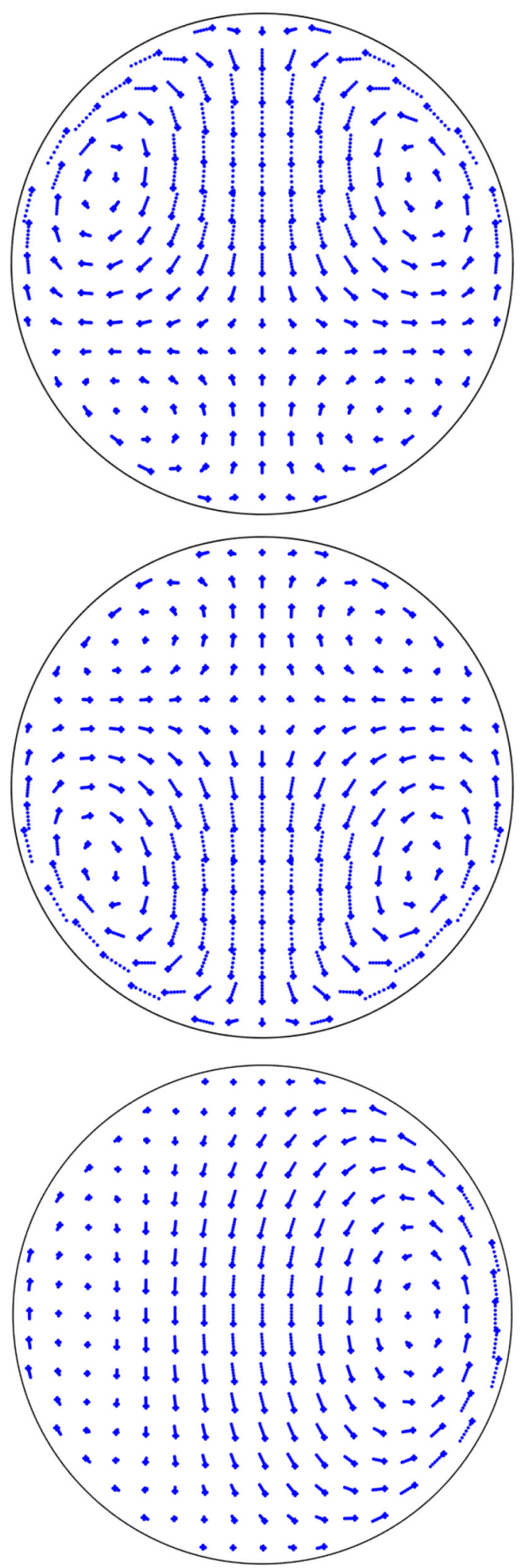

FIG. 3. Upper panel: Internal flow field of a liquid squirmer with pusher characteristics, obtained from the LB simulation with a surface velocity pattern according to Eq. (9) with $B_{2} / B_{1}=-3$. Middle panel: Flow field obtained for $B_{2} / B_{1}=+3$, resulting in the flow field of a puller. Lower panel: Flow field obtained from an asymmetric surface velocity pattern according to Eq. (10) with $c=1 / 2$. Shown is the section along the plane corresponding to $\phi=\frac{\pi}{2}, \frac{3 \pi}{2}$. 
surface velocity $v_{\theta, \phi}$ could be

$$
v_{\theta, \phi}=B_{1}(1+c \sin \phi) \sin \theta .
$$

The coefficient $c$ determines how pronounced the asymmetry is; an example with $c=1 / 2$ is shown in Fig. 3 (lower panel).

The above described examples demonstrate that we can obtain for our model droplet a reliable description of the internal flow field for an arbitrary velocity pattern in the droplet surface. A drawback of this approach is that the surface flow itself is not part of the simulation but a prescribed boundary condition. Thus, we cannot simulate phenomena which involve a change of the surface flow resulting, e.g., from changes in the surfactant concentration in the surrounding aqueous phase. However, for the study of the internal structure of a single self-propelling nematic droplet, our simple approach might be useful.

\section{DIRECTOR FIELD IN SELF-PROPELLING DROPLETS}

A description of the nematic director field can be made in terms of the unit vector $\vec{n}$ and the scalar order parameter $q$ ( $q=0$ : isotropic liquid, $q=1$ : perfect parallel orientational order). The vector approach neglects the physical equivalence of states with $\vec{n}$ and $-\vec{n}$ and has shortcomings in the description of topological defects [25]. A better description is provided by a tensor field $\mathbf{Q}(\vec{r})$. The tensor order parameter $\mathbf{Q}$ is defined as [26]

$$
Q_{\alpha \beta}=q\left(n_{\alpha} n_{\beta}-\frac{1}{3} \delta_{\alpha \beta}\right)
$$

its largest eigenvalue equals $\frac{2}{3} q$, and the corresponding eigenvectors are $\vec{n}$ and $-\vec{n}$.

Most nematic liquid crystals show the so-called flow aligning behavior, i.e., in a shear flow, the director aligns approximately (the deviation is of the order of $10^{\circ}$ ) along the flow direction. Few compounds do not show flow aligning but instead an unstable alignment ("tumbling") in a shear flow [27]. The coupling between flow and orientation works also in the opposite direction: When the orientation of $\vec{n}$ is changed, e.g., by the application of an electric field in a liquid crystal display, a flow ("backflow") in the material is induced [28]. Also, it is known for a long time that the effective viscosity in a shear flow depends on the orientation of $\vec{n}$ [29]. A first theoretical description of the mutual coupling between flow, director field, and viscosity was proposed by Ericksen [30] and Leslie [31,32] who used the vector description of the director field. Theoretical models of nematic hydrodynamics based on the tensor description were presented by Beris and Edwards [33,34] and Qian and Sheng [35]. LB models based on these theories have been developed by Care et al. [36,37] and Denniston et al. [38,39]. Recently, a multiparticle collision dynamics model, based on the Qian-Sheng theory, has been presented [40]. LB models, often in conjunction with finite difference schemes, have been applied to active nematic liquid crystals [41,42], nematic microfluidics [43-45], nematic colloids $[46,47]$, and other liquid crystal phases like cholesteric and blue phases [48,49]. In the following, we use a simplified version of the LB model described by Denniston et al. $[38,39]$ which is based on the Beris-Edwards theory. We first give a brief summary of the main equations of this theory.
In the Beris-Edwards framework, the evolution of the nematic tensor order parameter $\mathbf{Q}$ in the presence of a flow $\vec{v}$ is given by

$$
\left(\partial_{t}+\vec{v} \cdot \vec{\nabla}\right) \mathbf{Q}=\Gamma \mathbf{H}+\mathbf{S} .
$$

Here $\mathbf{H}$ is the molecular field which drives the system towards equilibrium and $\Gamma=2 q^{2} / \gamma_{1}$ where $\gamma_{1}$ is the rotational viscosity of the nematic liquid dystal $[38,39]$. $\mathbf{H}$ is obtained from the free energy $F$ of the system:

$$
\mathbf{H}=-\frac{\delta F}{\delta \mathbf{Q}}+\frac{\mathbf{I}}{3} \operatorname{Tr}\left(\frac{\delta F}{\delta \mathbf{Q}}\right) .
$$

For the free energy $F$, a standard Landau-de Gennes functional is used:

$$
\begin{aligned}
F= & \int\left[\frac{A_{0}}{2}\left(1-\frac{\gamma}{3}\right) \operatorname{Tr}\left(\mathbf{Q}^{2}\right)-\frac{A_{0} \gamma}{3} \operatorname{Tr}\left(\mathbf{Q}^{3}\right)\right. \\
& \left.+\frac{A_{0} \gamma}{4}\left[\operatorname{Tr}\left(\mathbf{Q}^{2}\right)\right]^{2}+\frac{L}{2}(\nabla \mathbf{Q})^{2}\right] d V .
\end{aligned}
$$

The first three terms describe a first-order transition, controlled by the parameter $\gamma$, of a generic liquid crystal $\left(\gamma>\frac{8}{3}\right.$ : nematic, $\gamma<\frac{8}{3}$ : isotropic phase) and the coefficient $A_{0}$ sets the bulk energy density of the liquid crystal. The last term gives the elastic energy where $L=K /\left(2 q^{2}\right)$ with $K$ being the mean of the three Frank elastic constants (one-constant approximation). With $F$ defined as above, we have for the molecular field $\mathbf{H}$ :

$$
\begin{aligned}
\mathbf{H}= & -A_{0}\left(1-\frac{\gamma}{3}\right) \mathbf{Q}+A_{0} \gamma\left(\mathbf{Q}^{2}-\frac{\mathbf{I}}{3} \operatorname{Tr} \mathbf{Q}^{2}\right) \\
& -A_{0} \gamma \mathbf{Q} \operatorname{Tr} \mathbf{Q}^{2}+L \nabla^{2} \mathbf{Q} .
\end{aligned}
$$

The second term on the right-hand side of Eq. (12), $\mathbf{S}$, describes the change of $\mathbf{Q}$ by the flow velocity gradient tensor $W_{\alpha \beta}=\partial_{\beta} v_{\alpha}$. Denoting the symmetric and antisymmetric part of $\mathbf{W}$ as $\mathbf{D}=\left(\mathbf{W}+\mathbf{W}^{T}\right) / 2$ and $\boldsymbol{\Omega}=\left(\mathbf{W}-\mathbf{W}^{T}\right) / 2, \mathbf{S}$ is given as

$$
\begin{aligned}
\mathbf{S}= & (\xi \mathbf{D}+\boldsymbol{\Omega})(\mathbf{Q}+\mathbf{I} / 3)+(\mathbf{Q}+\mathbf{I} / 3)(\xi \mathbf{D}-\mathbf{\Omega}) \\
& -2 \xi(\mathbf{Q}+\mathbf{I} / 3) \operatorname{Tr}(\mathbf{Q W}) .
\end{aligned}
$$

The factor $\xi$ depends on the shape of the nematic molecules and determines whether the material shows tumbling or flow aligning and, in the latter case, the equilibrium angle in a shear flow.

For the implementation of the LB model, we follow Refs. [38,39]. In order to describe the tensor field $\mathbf{Q}(\vec{r})$, a second set of distribution functions $\mathbf{G}_{i}$ is assigned to each grid node:

$$
\mathbf{Q}=\sum_{i} \mathbf{G}_{i}
$$

The evolution of the distribution functions $\mathbf{G}_{i}$ follows analogous to Eq. (4) with collision operators $\mathbf{C}_{i}=\frac{\Delta t}{\tau_{G}}\left(\mathbf{G}_{i}-\mathbf{G}_{i}^{\mathrm{eq}}\right)$ and coupling terms $\mathbf{M}_{i}$ which describe the influence of the flow field and the molecular field on $\mathbf{Q}$ :

$$
\mathbf{G}_{i}\left(\vec{r}+\vec{c}_{i} \Delta t, t+\Delta t\right)=\mathbf{G}_{i}(\vec{r}, t)-\mathbf{C}_{i}(\vec{r}, t)+\mathbf{M}_{i} .
$$


According to Ref. [39], the equilibrium distribution functions $\mathbf{G}_{i}^{\mathrm{eq}}$ are given as

$$
\mathbf{G}_{i}^{\mathrm{eq}}= \begin{cases}\mathbf{Q}\left[1-\frac{2(\vec{v} \cdot \vec{v})}{3}\right] & \text { for } \quad i=0 \\ \mathbf{Q}\left[\frac{\vec{v} \cdot \vec{c}_{i}}{3}+\frac{\left(\vec{v} \cdot \vec{c}_{i}\right)^{2}}{2}-\frac{\vec{v} \cdot \vec{v}}{12}\right] & \text { for } \quad i=1, \ldots, 6, \\ \mathbf{Q}\left[\frac{\vec{v} \cdot \vec{c}_{i}}{24}+\frac{\left(\vec{v} \cdot \vec{c}_{i}\right)^{2}}{16}-\frac{\vec{v} \cdot \vec{v}}{24}\right] & \text { for } \quad i=7, \ldots, 14\end{cases}
$$

and the coupling terms $\mathbf{M}_{i}$ are

$$
\mathbf{M}_{i}=\left\{\begin{array}{lll}
(\Gamma \mathbf{H}+\mathbf{S})\left(\frac{1}{15}+\frac{\vec{v} \cdot \vec{c}_{i}}{3}\right) & \text { for } \quad i=0, \ldots, 6 \\
(\Gamma \mathbf{H}+\mathbf{S})\left(\frac{1}{15}+\frac{\vec{v} \cdot \vec{c}_{i}}{24}\right) & \text { for } \quad i=7, \ldots, 14
\end{array}\right.
$$

These expressions result from similar expansions and constraints as mentioned for Eq. (6), details are given in [39].

So far we have considered only how the combination of flow field and molecular field would influence the $\mathbf{Q}(\vec{r})$ field. In order to take into account the inverse coupling, i.e., the influence of the anisotropic viscosity and of backflow effects on the $\vec{v}(\vec{r})$ field, we would have to calculate the stress tensor of the nematic liquid and implement it into the LB model. The calculation and implementation are described in [38,39], and one consequence is a modification and extension of Eq. (6). However, because of the considerably smaller computational effort we tried as a first approach to treat the nematic liquid crystal as a Newtonian fluid with isotropic viscosity without any backflow effects. It is obvious that this is a strong simplification but the results seem to indicate that the $\mathbf{Q}(\vec{r})$ field in the self-propelling droplet is surprisingly well described by this simple approach. We come back to this point in the discussion section.

Standard ionic surfactants possessing a hydrophilic head and a long hydrophobic tail induce a perpendicular anchoring of the nematic director at the liquid crystal-aqueous interface [50]. As boundary condition for $\mathbf{Q}$ we thus implement strong perpendicular anchoring, i.e., at all nodes located at the boundary $\mathbf{Q}$ is reset after each time step to its initial value corresponding to a director orientation perpendicular to the boundary. The results presented in the following were obtained for LB simulations of a spherical model droplet (experimental diameter $50 \mu \mathrm{m}$ ), which is placed into a cubic grid with $k^{3}$ nodes (for most runs we set $k=64$, in a few cases we used $k=128$ ). For the mapping between physical and simulation units we use an earlier experiment [51] in which the flow of 5CB through a microfluidic channel was studied. More details are given in the Supplemental Material [52].

In a resting nematic droplet, the structure of the director field is determined only by the nematic elasticity, the size of the droplet, and the anchoring condition at the droplet surface [54-56] resulting, for larger droplets and strong perpendicular anchoring, in a simple radial director field with a topological point defect of strength +1 at the center. We use this structure (shown in Fig. 5, upper panel) as start configuration and study first the effect of a symmetric flow field (Fig. 1), corresponding to a neutral squirmer, on the structure of the director field in our self-propelling nematic model droplet. When the surface flow is switched on, the internal convection develops, and the structure of the $\vec{n}$ field evolves in the concurrent influences of elastic interactions and flow-aligning effects. The torque on $\vec{n}$ in a shear flow is proportional to the magnitude of the
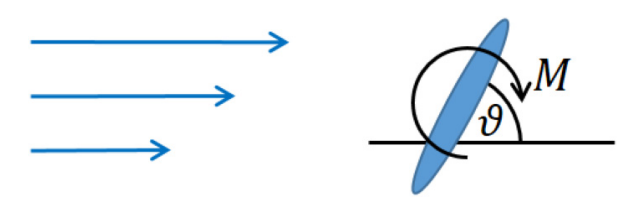

FIG. 4. Flow alignment in a shear flow (blue arrows): the nematic director (blue ellipse) experiences a torque $M=\left(\alpha_{2} \sin ^{2} \vartheta-\right.$ $\left.\alpha_{3} \cos ^{2} \vartheta\right)|\nabla v|$ [53], $M$ vanishes for $\vartheta=\arctan \sqrt{\alpha_{3} / \alpha_{2}} \approx 10^{\circ}$ for most compounds $\left(\alpha_{2,3}\right.$ are viscosity coefficients of the EricksenLeslie theory, which can be related to the scalar order parameter $q$ and the quantities $\xi$ and $\Gamma$ of the Beris-Edwards framework [38]).

flow velocity gradient, $|\vec{\nabla} v|$, and depends on the orientation of $\vec{n}$ with respect to $\vec{\nabla} v$; see Fig. 4 . As shown in Fig. $2, v$ varies, in the equatorial plane of our model droplet, as a simple parabola, i.e., $|\vec{\nabla} v|$ goes to zero at the droplet center and is maximum at the surface. Also, considering the initial $\vec{n}$ field (Fig. 5, upper panel) and the applied flow field (Fig. 1), we note that the deviations between the initial orientation of $\vec{n}$ and the expected orientation of $\vec{n}$ in the flow field (Fig. 4) are larger in the lower half-sphere $(z<0)$ of our model droplet compared to the upper $(z>0)$ half-sphere. Taking additionally into account that the orientation of $\vec{n}$ is held constant at the surface (strong anchoring), we expect the largest flow aligning effects in regions in the lower half-sphere and at some distance from the center and the surface. Figure 5 (middle panel) shows an intermediate state of the LB simulation confirming these expectations.

The flow-induced changes of the orientation of $\vec{n}$ result in a shift of the point defect along the central symmetry axis in the downstream direction. Because flow gradients are small in the central region, this shift proceeds slower than the changes in regions closer to the surface. However, the LB simulation reaches finally a steady state in which the flow aligning effects are balanced by the elastic interactions (Fig. 5, lower panel). The position of the point defect is now near the stagnation point at the leading pole of the self-propelling droplet, in accordance with the experimental observation of nematic droplets self-propelling in capillaries or microfluidic channels (which enforce a straight motion of the droplets) $[3,8]$. Structural changes of nematic director fields by convective flows have been observed also in cylindrical droplets, in which the convection was induced by temperature gradients [57], electric fields [58], or the advection of the droplet in microfluidic channels [59].

The drawings in Fig. 5 show only every fourth of 64 nodes along one dimension. Looking at the director field around the defect in higher resolution, it seems that the point defect has spread into a topological equivalent loop of a line defect, a disclination line of strength $+\frac{1}{2}$ (Fig. 6). The fine structure around the core of a radial point defect and its possible transformation into a disclination loop or other structures have been subjects of several studies (see Ref. [60] and references therein). However, the spatial resolution of our simulation ( $\Delta x$ in physical units is $50 / 64 \mu \mathrm{m}$ ) is too low to obtain reliable results about the defect core. The reason for the appearance of the disclination loop in the simulation is probably due to shortcomings of the numerics: In grid-based simulations the 

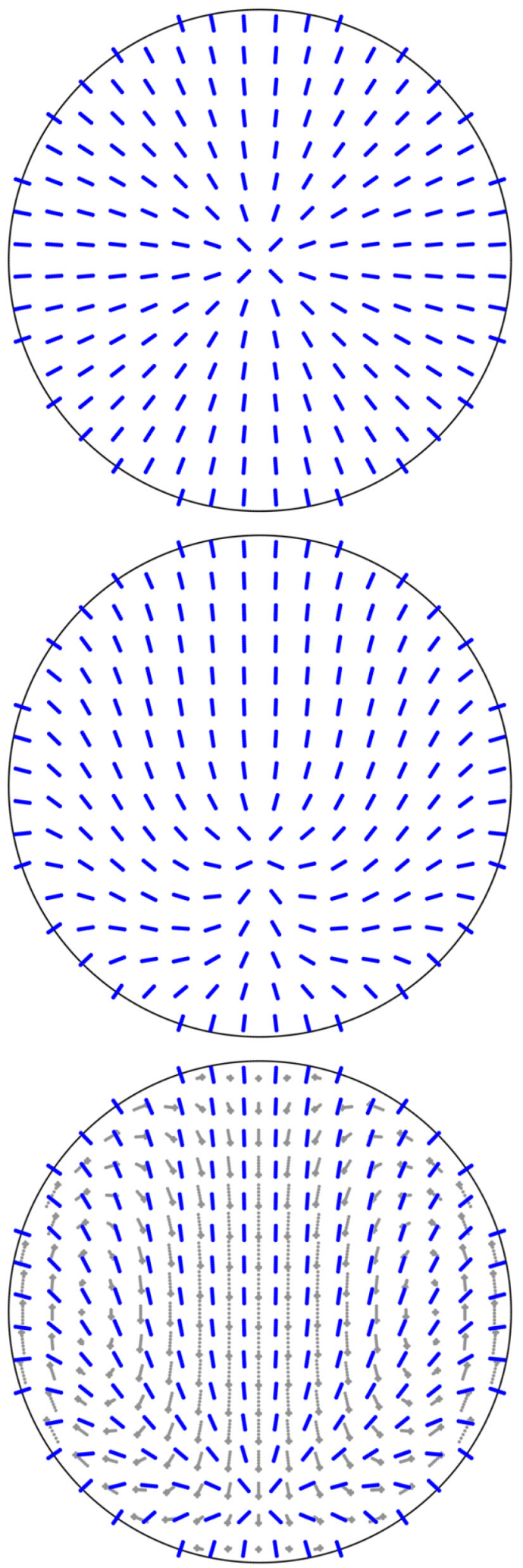

FIG. 5. Upper panel: Radial director field used as start configuration for the LB simulation; blue rods indicate the orientation of the local director. Middle panel: Intermediate structure of the director field, obtained after one quarter of the time span necessary to reach the steady state. Lower panel: Final structure (steady state) of the director field in a symmetric flow field (gray arrows, Fig. 1).

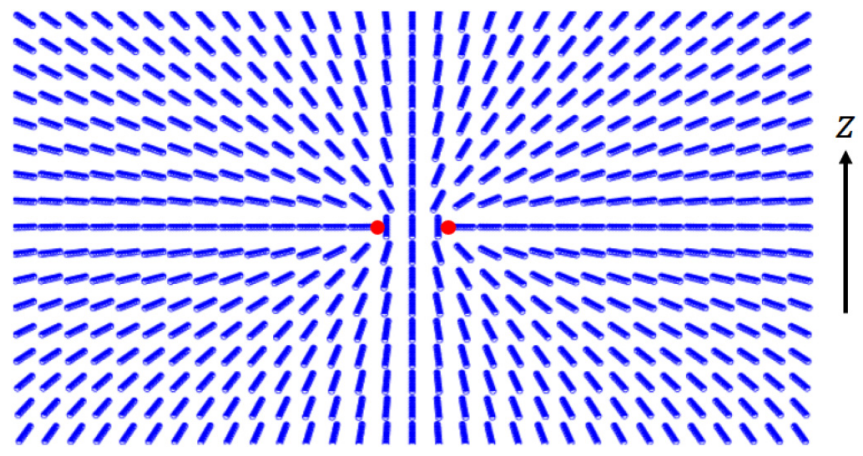

FIG. 6. Director field of the defect region in the steady state (same data as in Fig. 5, lower panel) in higher resolution. The point defect is replaced by a small disclination loop, which is located in the plane perpendicular to the $z$ axis; red dots indicate the intersection of the disclination loop with the plane of the image.

numerical value of the elastic energy is in regions around defects always smaller than the real value. This could be compensated by adaptive grid techniques [61] which are beyond the scope of the present study. However, the numerical error is for a line defect considerably larger than for a point defect resulting in a misleadingly low elastic energy of the line defect. Thus, in our simulated director fields the point defect is always replaced by a small disclination loop.

In addition to the simple symmetric flow field shown in Fig. 1 we have also studied the effect of the flow patterns shown in Fig. 3. Figure 7 shows the internal structure (the steady state at the end of the LB simulation) of self-propelling nematic droplets possessing pusher or puller characteristics. As to be expected, the largest changes in the $\vec{n}$ field, with respect to the initial configuration (Fig. 5, upper panel), occur for the pusher in the rear and for the puller in the front half of the droplet. The central point defect is shifted in the pusher only by a small amount towards the internal stagnation point of the flow pattern, whereas it is shifted to the leading pole of the puller.

When nematic droplets self-propel in capillaries or microfluidic channels with a width comparable to the droplet diameter, they move inevitably on a straight trajectory and the point defect retains its position at the leading pole of the droplet. In a two- or three-dimensional environment, however, it is observed that the defect is advected away from the stagnation point at the front of the droplet, i.e., it adopts a position somewhere between the leading pole and the equator. Also, the surface flow pattern is asymmetric and the trajectory of the droplet becomes curved [8]. We have thus studied how an asymmetric flow pattern as shown in the lower panel of Fig. 3 affects the director field in our model droplet. The start configuration of the LB simulation is again a simple radial director field as shown in Fig. 5 (upper panel). The first structural changes are similar to those observed for the symmetric flow pattern: the central defect is shifted towards the leading pole of the droplet. Near the surface, however, the position of the defect is deflected towards the side of the maximum amplitude of the surface flow. Finally, a steady state is reached (see Fig. 8) with the defect being located near 

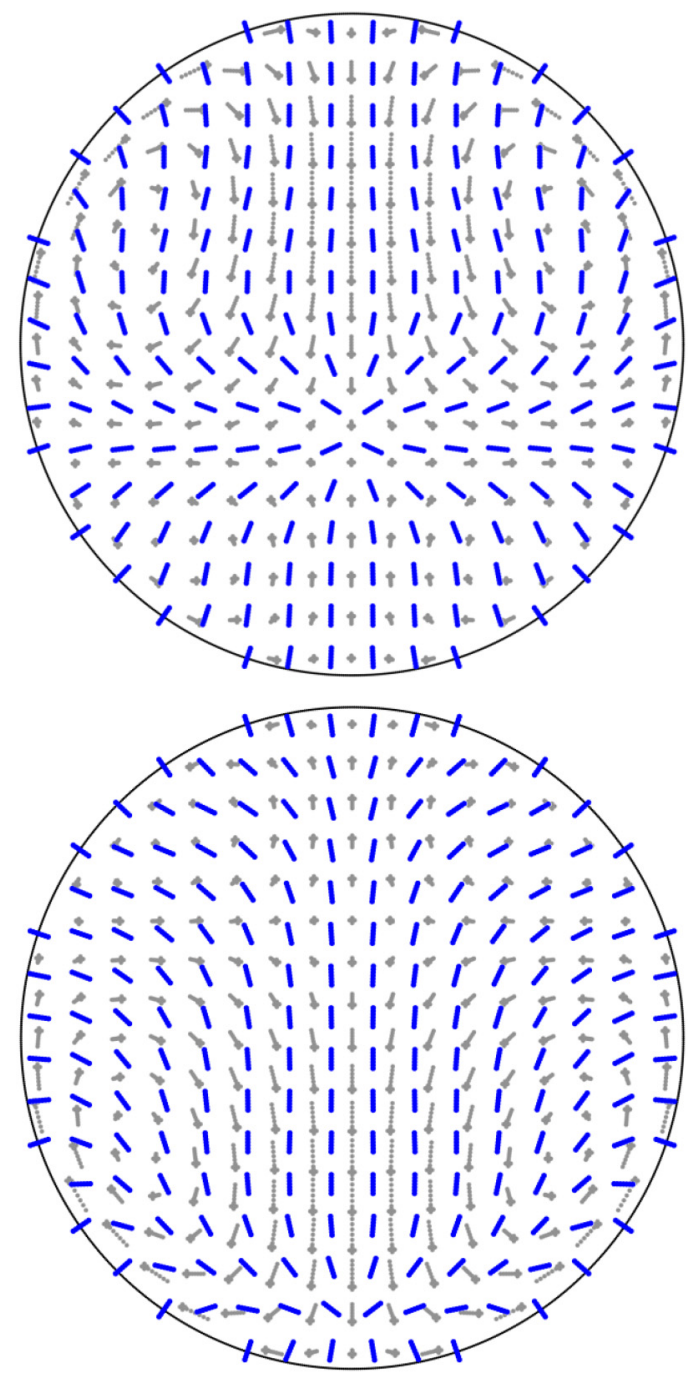

FIG. 7. Upper panel: Director field (steady state) resulting from the internal flow field (gray arrows, Fig. 3, upper panel) of a selfpropelling droplet with pusher characteristics. Lower panel: Director field (steady state) resulting from the internal flow field (gray arrows, Fig. 3, middle panel) of a puller.

the surface and with a deflection angle of $45^{\circ}$ to $60^{\circ}$ from the leading pole, in accordance with the experimental observations [8]. The broadening of the point defect into a disclination loop is more pronounced than for the symmetric flow pattern but, as described above, this may be due to the numerical underestimation of the elastic energy of a disclination loop compared to a point defect.

The above described results confirm the experimental observation of an advection-like behavior of the central point defect, which is shifted, depending on the flow pattern, either to the leading pole of the droplet or to a position between the leading pole and the equator on the side of the maximum surface flow. If the final position of the defect in the flow field is known from the experiment, we can use a kind of shortcut to obtain the final structure of the director field in the internal flow field of the whole droplet: The first step of this approach is to set the defect position "manually" as a part of the boundary conditions. This is done by defining, around

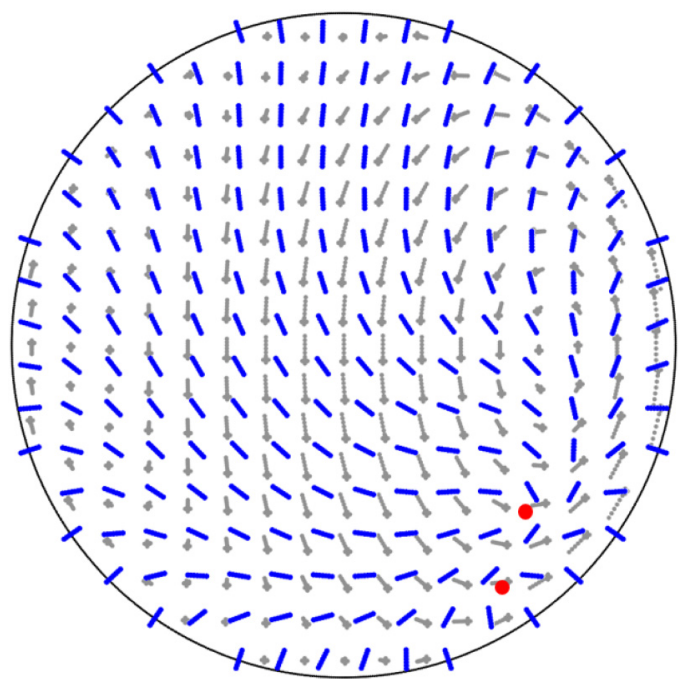

FIG. 8. Director field (steady state) resulting from an asymmetric internal flow field (gray arrows, Fig. 3, lower panel). The point defect has broadened to a disclination loop which is located in a plane perpendicular to the image, red dots indicate the intersection of the disclination loop with the plane of the image.

the known defect position, a small spherical volume in which the director field is kept fixed in a radial configuration. The next step is to minimize the elastic energy which results from the perpendicular anchoring at the droplet surface and the position of the defect. Because flow effects are not considered at this stage, the energy minimization can be done, e.g., by the fast $Q$-tensor method [62,63]. The obtained director field is then used as start configuration for the LB simulation which arrives faster at a steady state, saving at least $50 \%$ computation time compared to the case where the defect position is not held constant. Figure 9 illustrates this approach: the upper panel shows the director field obtained by setting the defect position near the leading pole and minimizing subsequently the elastic energy, the lower panel shows the steady state of the LB simulation, for which the structure shown in the upper panel was used as start configuration. The final structure is nearly the same as in the case where the defect position was not kept fixed (Fig. 5, lower panel) but the computation time is only one third of that case.

\section{JONES MATRIX CALCULATIONS}

To what extent correspond the simulated structures described in Sec. III to the structures of real self-propelling nematic droplets? An important tool to study the structure of liquid crystalline samples is the polarizing microscope. The birefringence of the liquid crystal leads to characteristic patterns and textures and some features, e.g., the presence of topological defects, can be directly determined from microscopy images. A detailed comparison of a given model structure with an experimental structure is provided by the Jones matrix method [64], which enables the calculation of microscopy images of a simulated structure. The numerical micrographs can be directly compared with micrographs of the real sample thereby probing the reliability of the simulated structure. 

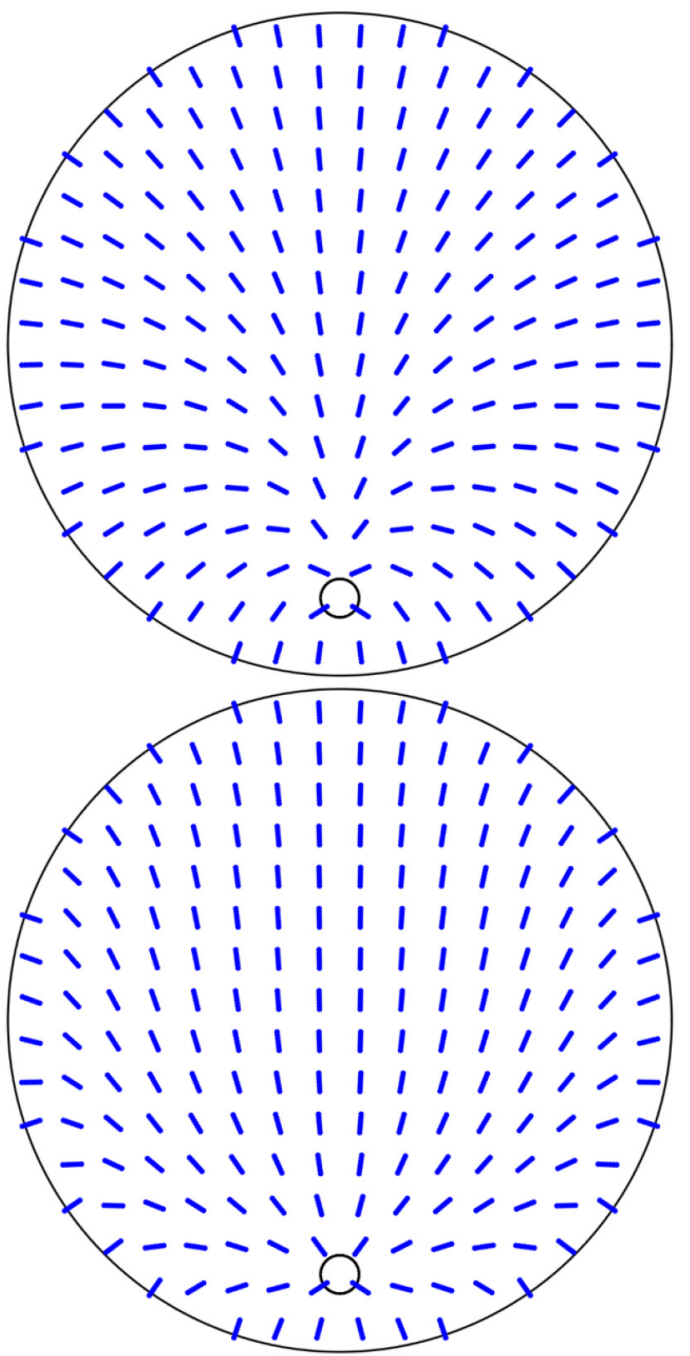

FIG. 9. Upper panel: Director field obtained by setting the defect position near the lower surface of the droplet and subsequent minimization of the elastic energy. The small black circle indicates the region in which the director orientation is kept constant in a radial configuration. Lower panel: Director field (steady state) resulting from a symmetric internal flow field (Fig. 1). The structure shown in the upper panel was used as start configuration and the defect position was held constant.

The Jones matrix formalism relates the electric field $\vec{E}_{\text {out }}$ of a light wave which has transmitted an optical system to the electric field $\vec{E}_{\text {in }}$ of the incident light wave. The incident (monochromatic) light wave is assumed to possess a welldefined polarization described by amplitude $|E|$ and phase $\delta$ of two orthogonal components, e.g., for a light wave propagating along $z$ :

$$
\begin{aligned}
\vec{E}_{\mathrm{in}} & =\left(\begin{array}{l}
E_{x} \\
E_{y}
\end{array}\right) \quad \text { with } \\
E_{x, y} & =\left|E_{x, y}\right| \exp \left(\iota \delta_{x, y}\right) .
\end{aligned}
$$

The electric field of the transmitted light is then obtained as

$$
\vec{E}_{\text {out }}=\mathbf{J} \vec{E}_{\text {in }}
$$

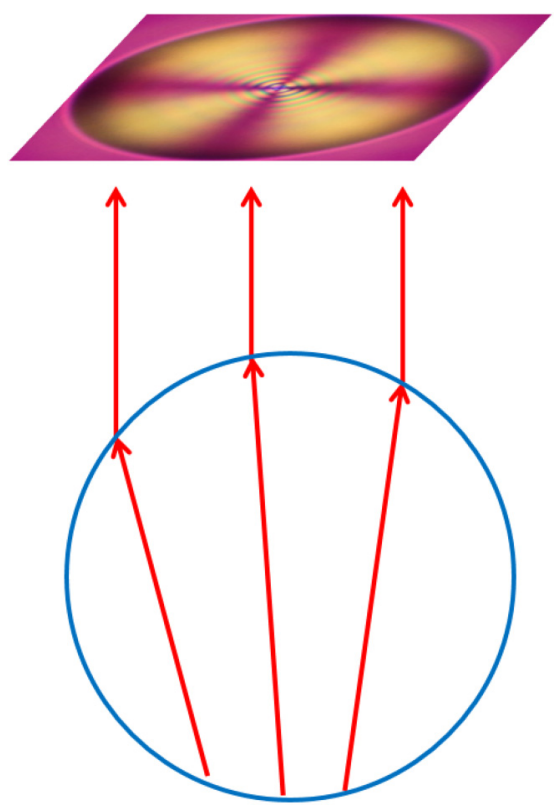

FIG. 10. Schematic illustrating the refraction of light rays taken into account for computing the numerical micrograph.

where the $2 \times 2$ matrix $\mathbf{J}$ is determined by the structure and optical properties of the transmitted system, which usually consists of the sample and some optical elements (polarizers, wave plates, etc.). The Jones matrix $\mathbf{J}$ of the complete transmitted system is obtained as the product of the matrices of all transmitted elements, e.g., for a typical configuration of a polarizing microscope with polarizer $P$, sample $S$, wave plate $W$, and analyzer $A$ :

$$
\mathbf{J}=\mathbf{J}_{A} \mathbf{J}_{W} \mathbf{J}_{S} \mathbf{J}_{P}
$$

The Jones matrix of the sample, $\mathbf{J}_{S}$, is obtained as a product $\mathbf{J}_{S}=\prod_{i} \mathbf{J}_{S, i}$ where the matrices $\mathbf{J}_{S, i}$ correspond to small voxels into which the sample is divided along the path of the transmitting light. Within each voxel the optical properties, which depend on the orientation of the local director, are assumed as constant. Explicit expressions for the matrices of the optical elements and the sample voxels can be found in Ref. [65] and are given in the Supplemental Material [52].

Usually, for samples with plane boundaries, parallel light rays are assumed which enter and leave the sample perpendicular to the boundaries. For spherical samples (droplets) refraction at the surface can be neglected if the difference between the refractive indices of sample and environment is small as is the case, e.g., for nematic droplets dispersed in a polymer matrix [66,67] or for lyotropic liquid crystals [68]. A more elaborated Jones matrix method for liquid crystal droplets, taking into account the numerical aperture of the microscope optics, was proposed by Mur et al. [69]. In the present study, we take refraction at the droplet surface into account but we do not consider the numerical aperture of the microscope, i.e., we assume that the image is formed by parallel rays which arrive perpendicular to image plane but travel with different angles through the droplet (Fig. 10). The refraction angles at the droplet surface are calculated assuming a mean refractive index of the liquid crystal, neglecting the 
different refraction of ordinary and extraordinary rays. The birefringence of the liquid crystal, however, enters through the phase difference between ordinary and extraordinary rays which is calculated using the refractive index values of $5 \mathrm{CB}$ reported in [70].

The calculated director field resulting from the LB simulation gives the director orientation at each grid node. Because the light ray transmits the droplet at some angle to the coordinate axis, the director orientation at the sampling points along the ray is approximated as described in [69]. For each pixel of the numerical image, the transmitted field $\vec{E}_{\text {out }}$ is calculated as a function of light wavelength $\lambda$ in the range between $390 \mathrm{~nm}$ and $790 \mathrm{~nm}$, and we obtain finally an intensity spectrum $I(\lambda)$ for each pixel. The last step is then the transformation of the intensity spectrum to a color as seen by the human eye. For this purpose, the intensity spectrum is weighted by three color matching functions which reflect the wavelength sensitivity of the optical receptors in the eye. We use the color matching functions $\tilde{x}(\lambda), \tilde{y}(\lambda)$, and $\tilde{z}(\lambda)$ of the CIE 1931 standard observer [71] in order to obtain the $X, Y$, and $Z$ coordinates in the CIE XYZ color space:

$$
X=\int I(\lambda) \tilde{x}(\lambda) d \lambda
$$

(equations for $Y$ and $Z$ read analogously). Comparing the colors of an experimental image with those of a calculated image is somewhat ambiguous because there are lot of factors which influence the experimental recording of a color, e.g., the light source and optical elements, the camera that was employed, the software which is used to display or print the image, etc. We found it useful to consider the magenta background color that is obtained in a polarizing microscope with a wave plate for optically isotropic samples (or without any sample), and we vary the magenta color which is computed by the Jones matrix formalism until it coincides, as judged by the eye, with the experimental magenta. This variation is done by adjusting the spectrum of the incident light $\vec{E}_{\text {in }}$ : we assume a shape of the spectrum corresponding to the black body radiation and vary the temperature.

A basic test of our implementation is the comparison with an experimental micrograph obtained for a liquid crystal sample the structure of which is well known. Figure 11 (upper panel) shows the polarized micrograph (crossed polarizers with diagonal wave plate) of a 5CB droplet floating in the surfactant solution. Because the surfactant concentration is below the threshold for self-propulsion, the droplet shows a simple radial director field with $\mathrm{a}+1$ point defect in its center. The lower panel of Fig. 11 shows the numerical micrograph calculated for a radial director field (see Fig. 5, upper panel). The good agreement between experimental and calculated image demonstrates the usefulness of our approach.

We first consider the case of the symmetric flow field (Fig. 1) which is present in droplets self-propelling in capillaries or microfluidic channels on a straight trajectory. Figure 12 shows an experimental micrograph of a $5 \mathrm{CB}$ droplet selfpropelling in a capillary, the point defect is located near the bottom of the droplet. When we calculate a numerical micrograph for the director field shown in the upper panel of Fig. 9 (defect position set near the bottom, elastic energy minimized, zero flow) we find only a poor agreement between
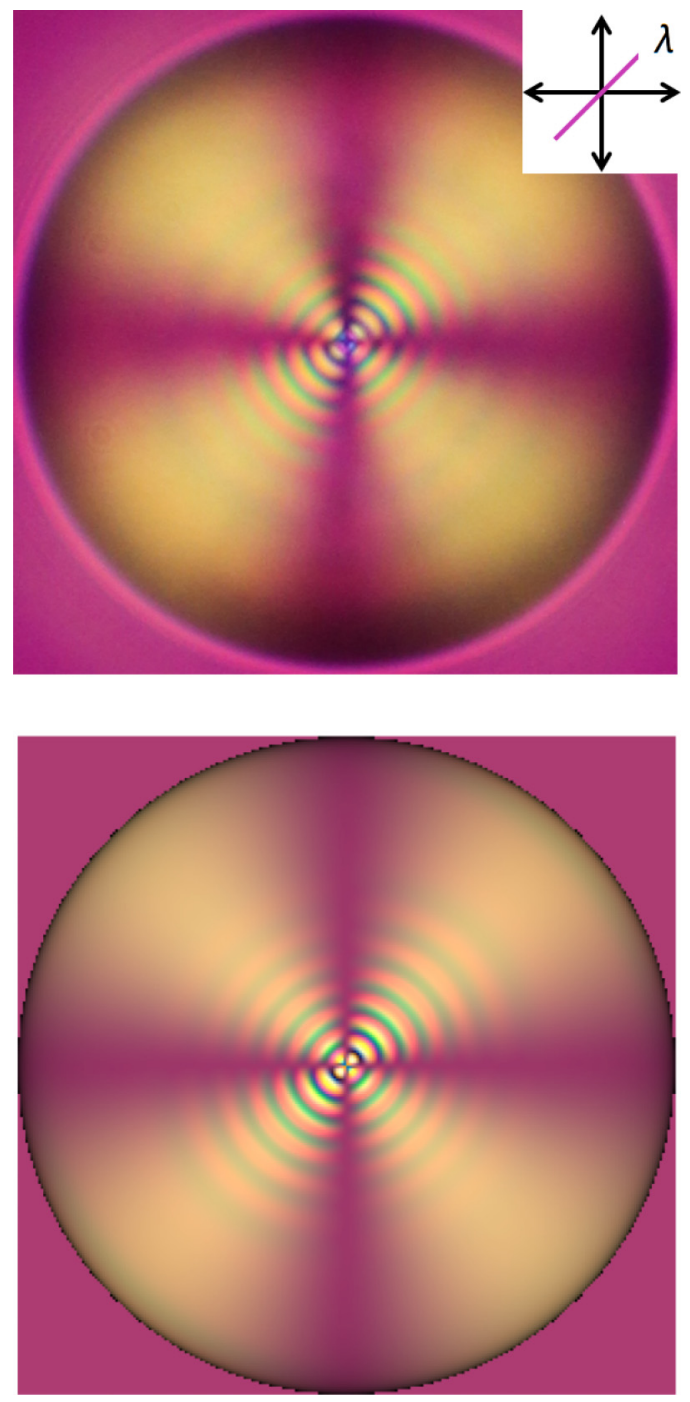

FIG. 11. Upper panel: Micrograph (crossed polarizers, as indicated in the top right corner, with diagonal wave plate) of a $5 \mathrm{CB}$ droplet (diameter $46 \mu \mathrm{m}$ ) floating in a surfactant solution inducing strong perpendicular anchoring at the droplet surface resulting in a radial director. Lower panel: Calculated micrograph for a model radial director field under coincident conditions (diameter, refractive indices, etc.).

experimental and numerical image (see Fig. 13). This is to be expected because that director field is based solely on the minimization of the elastic energy, without considering any flow effects.

However, we get better results if we add the internal convection to the simulation of the director field: Fig. 14 shows numerical micrographs for three different director fields obtained from LB simulations using as start configuration the zero-flow director field with fixed defect position (upper panel of Fig. 9). The simulations were run with different values of the amplitude $B_{1}$ of the surface flow field [see Eq. (1)] until the steady states were reached for which then the micrographs were calculated. The experimental and numerical micrographs possess three salient features: the interference pattern around the defect at the leading pole, the two larger colored regions near the equator, and the two smaller colored 


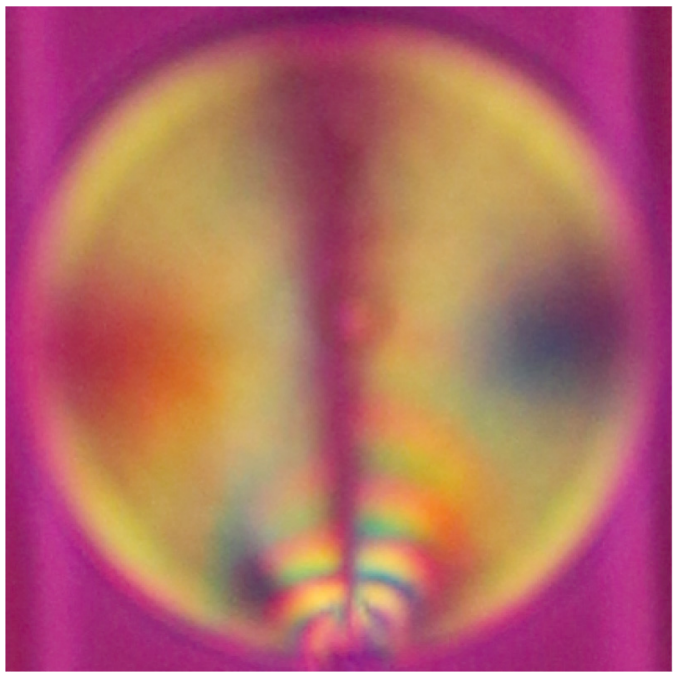

FIG. 12. Micrograph (crossed polarizers with diagonal wave plate, same orientation as shown in Fig. 11) of a 5CB droplet (diameter $50 \mu \mathrm{m}$ ) self-propelling in a cylindrical capillary. The droplet is moving downward, the point defect is located near the leading pole of the droplet.

spots, with swapped colors of the larger regions, at the sides of the interference pattern. The numerical micrograph displayed in the middle panel of Fig. 14 shows a fair agreement to the experimental image with regard to all three features. Using the mapping between LB and physical units provided in the Supplemental Material [52], the velocity of the internal flow in the center of the droplet is of the order of $20 \mu \mathrm{m} / \mathrm{s}$ which coincides well with the experimental observation.

We now turn to the case of the asymmetric internal flow field, which is present in self-propelling nematic droplets

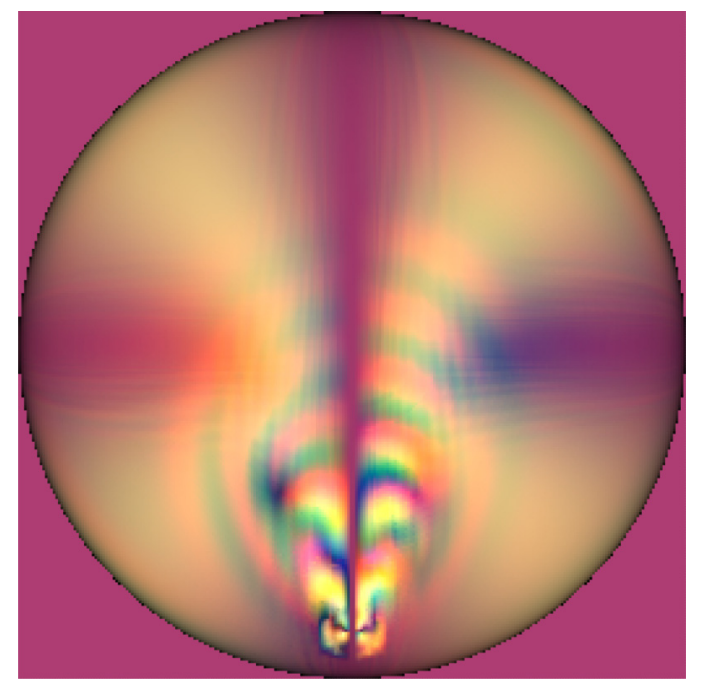

FIG. 13. Calculated micrograph for the model director field shown in the upper panel of Fig. 9. Because flow effects were not considered for that director field, there is only a poor agreement to the experimental image (Fig. 12).
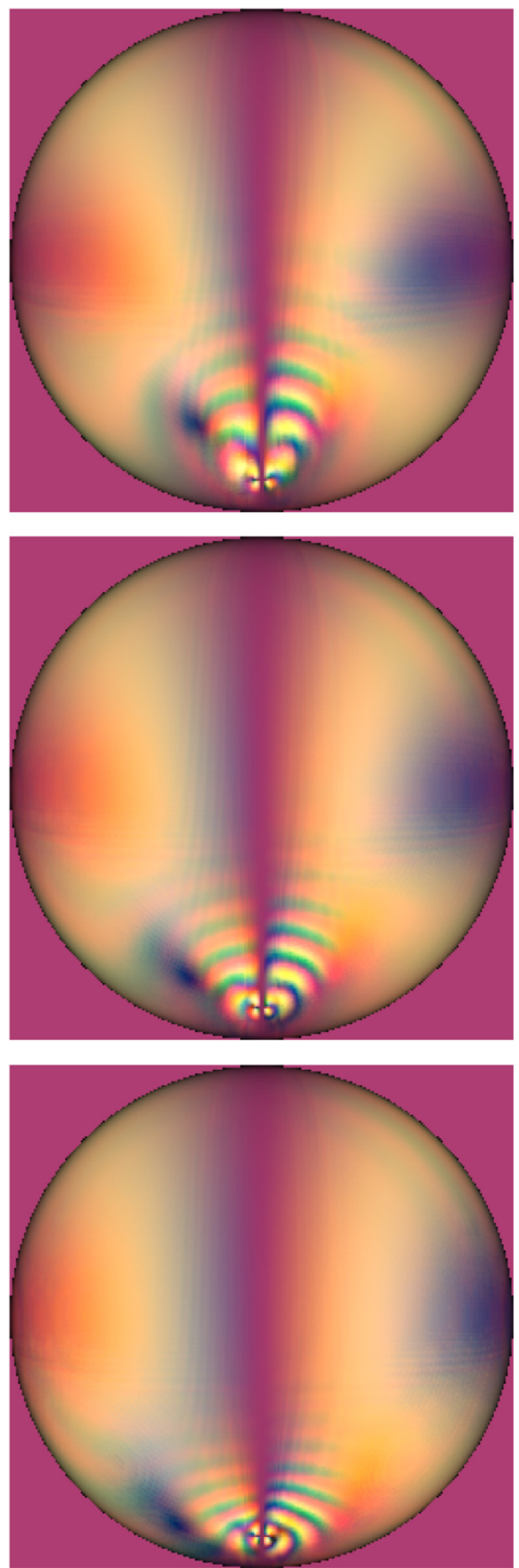

FIG. 14. Calculated micrographs for three model director fields obtained from LB simulations with three different amplitudes $B_{1}$ [Eq. (1)] of the surface flow field. With respect to the $B_{1}$ value for the upper panel, the value was increased by a factor of 2 for the middle panel and by a factor of 4 for the lower panel. 


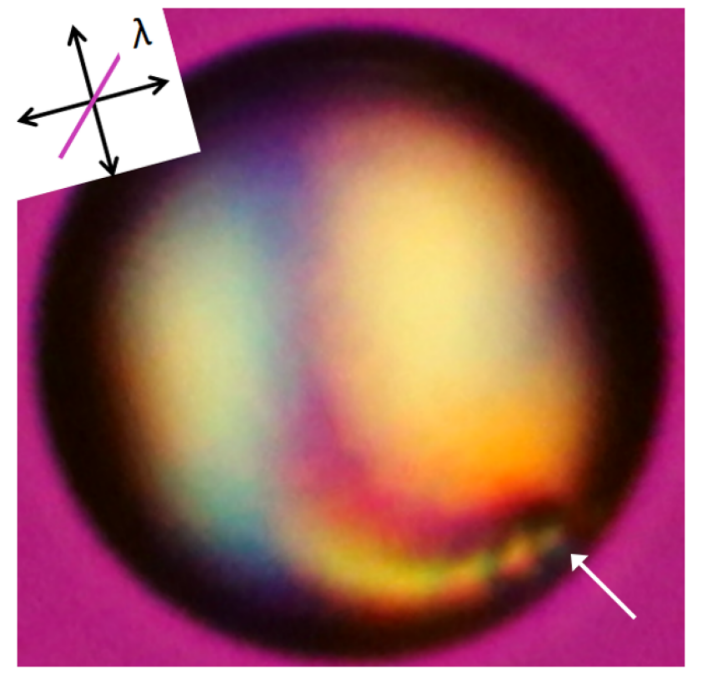

FIG. 15. Micrograph (crossed polarizers with diagonal wave plate, orientation as indicated in the top left corner) of a 5CB droplet (diameter $50 \mu \mathrm{m}$ ) self-propelling in a quasi-2D reservoir. The droplet is moving downward; the approximate position of the point defect is indicated by the white arrow.

which are not confined in a capillary but move in a quasi-2D or 3D reservoir. Figure 15 shows an experimental micrograph of a 5CB droplet self-propelling in a quasi-2D environment, the instantaneous motion direction is downward but the trajectory is curved to the left. The position of the point defect is near the droplet surface though not at the leading pole but deflected towards the equator by an angle of about $\pi / 4$. The surface flow and the resulting internal flow field is asymmetric as shown in the lower panel of Fig. 3.

In order to determine a director field structure that reproduces the experimental micrograph, we use the same approach as for the symmetric flow field. The defect is set to the position known from the experiment and the elastic energy is minimized under the condition of zero flow. The obtained director field (which is the same as shown in the upper panel of Fig. 9 but rotated counterclockwise by $\pi / 4$ ) is then used as start configuration for the LB simulation with an asymmetric surface flow pattern according to Eq. (10). As shown in Fig. 16, we can achieve a good agreement between calculated and experimental micrographs by varying the amplitude $B_{1}$ (see middle panel of Fig. 16). The director field structures, from which the micrographs shown in Fig. 16 were calculated, were obtained with a defect position that was fixed as part of the boundary conditions. Thus, the asymmetry in the flow field would not be necessary to advect the defect to a certain position and a symmetric flow field could have been used in the LB simulation. Nevertheless, comparing the results obtained with symmetric and asymmetric flow fields, we find that a certain degree of asymmetry in the flow field, described by the coefficient $c$ in Eq. (10), improves the conformance between calculated and experimental micrographs. In other words, the asymmetry in the flow field determines not only the defect position but influences also the whole director field of the droplet.
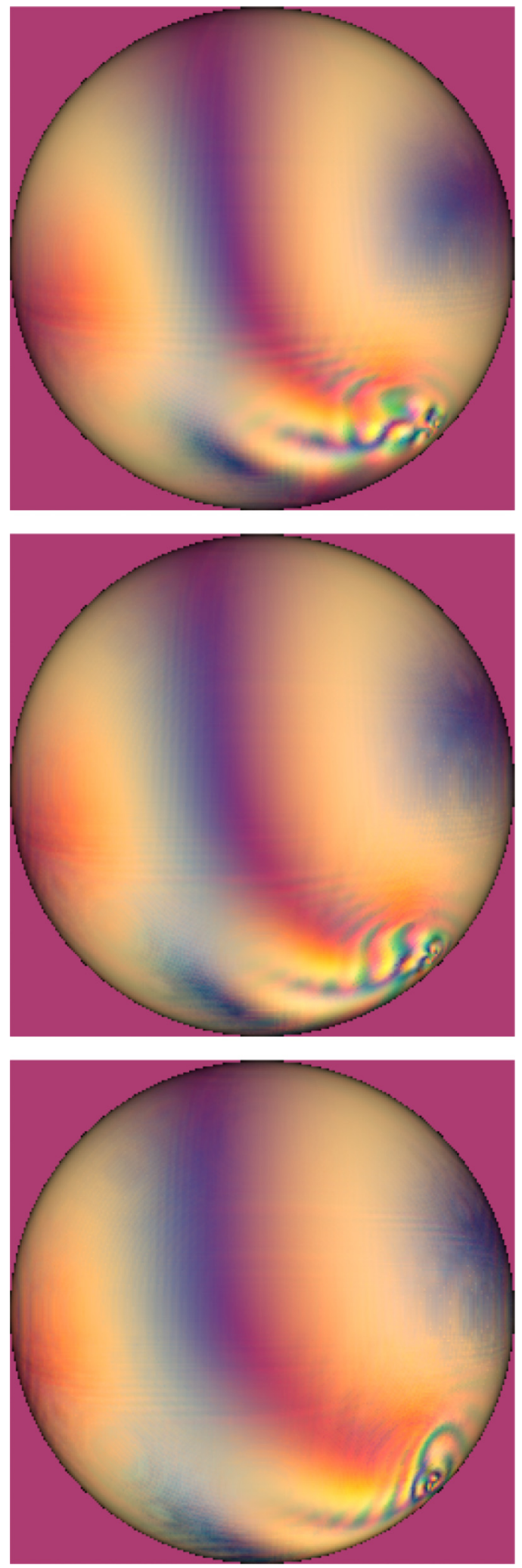

FIG. 16. Calculated micrographs (crossed polarizers with diagonal wave plate, orientation as indicated in Fig. 15) for three model director fields obtained from LB simulations with asymmetric surface flow patterns according to Eq. (10) with $c=1 / 4$ and different amplitudes $B_{1}$. With respect to the $B_{1}$ value for the upper panel, the value was increased by a factor of 2 for the middle panel and by a factor of 4 for the lower panel. 


\section{DISCUSSION AND CONCLUSION}

So far we have obtained the following results: The internal flow in a self-propelling droplet, driven by a Marangoni surface flow, can be described by a standard LB model with a spherical boundary being realized by an interpolation procedure [20]. The Marangoni surface flow can be implemented as a tangential boundary velocity pattern, appropriate variation of the surface flow pattern leads to symmetric or asymmetric convective or puller- or pusher-like flow patterns in the droplet. Concerning the nematic director field in the droplet, a simplified version of the LB model of Denniston et al. $[38,39]$, neglecting the anisotropic viscosity and the backflow coupling in the nematic phase, can sufficiently describe the flow-induced changes of the director field and the advection of the topological point defect. The final position of the defect, if it is known from the experiment, can be implemented also as an additional boundary condition, resulting in a considerably shorter computation time for obtaining the final steady-state structure. Jones matrix calculations enable an efficient check of the reliability of the computed structures.

With our current implementation of the LB simulation, several topics cannot be sufficiently addressed, e.g., the physical origin of the asymmetry of the flow field of droplets selfpropelling in a quasi-2D or 3D environment. It was argued [8], that the origin of the flow asymmetry is the structural asymmetry of the director field: The first step is a spontaneous, fluctuation-induced displacement of the point defect from the leading pole. Then, because of the lower viscosity around the defect, the flow becomes stronger in the droplet half in which the defect is located and the asymmetry of the whole flow field develops. The results of the present LB simulations show that the inverse order could be also true because an asymmetry in the flow field leads to an asymmetric position of the point defect. Probably, both effects play a role and form a positive feedback: the change of the defect position enhances the flow asymmetry which in turn increases the deflection of the defect. However, in order to study these effects numerically, two features have to be implemented in the LB framework: (1) the anisotropy of the nematic viscosity and (2) the surface flow should become part of the simulation instead of being a boundary condition. The anisotropic viscosity is necessary for the correct description of the flow field, especially near the point defect. The surface flow should be included in the LB simulation because the prescription of the surface flow as a boundary condition suppresses, at least to a certain degree, the influence of regions with different effective viscosities in the droplet which result from different director orientations.

We are currently conducting preliminary investigations of the role of backflow and anisotropic viscosity in the LB simulation of self-propelling nematic droplets. First results indicate that the inclusion of the backflow coupling has some influence on the intermediate states but only little influence on the steady state at the end of the simulation. Thus, if we are primarily interested in the steady-state structure of the director field in the droplet, characterized by an equilibrium between flow and elastic effects, our simple approach of prescribing a certain surface flow pattern and neglecting the anisotropy of the nematic viscosity seems to be sufficient.

The second point, the inclusion of the surface flow pattern into the LB simulation, would require a considerable extension of the LB framework and is left for future research. One approach could be to use a LB model for a binary system like two immiscible liquids [72,73]. Then, the nematic droplet would be simulated floating in the aqueous environment and the self-propulsion could be induced by a body force the direction of which is coupled to the flow field of the droplet. Under these conditions, the flow field in the droplet could develop independently of a certain velocity pattern prescribed in the droplet surface.

The use of LB models for two immiscible liquids could be also a first step for the simulation of self-propelling nematic shells [11]: The nematic model droplet, as described in the present paper, then would be a spherical reservoir in which a smaller aqueous droplet is floating and one could study the advection of the aqueous droplet in the nematic flow field and the mutual interaction between the flow fields of the shell and the internal droplet.

\section{ACKNOWLEDGMENTS}

We thank Stephan Herminghaus, Corinna C. Maass, Carsten Krüger, Babak V. Hokmabad, Kyle A. Baldwin, Jens I. Meyer, and Martin W. Wagner for stimulating discussions and/or sharing of experimental results. Financial support from the Deutsche Forschungsgemeinschaft (Grant no. BA 1048/8, priority program SPP 1726 Microswimmers) is gratefully acknowledged.
[1] C. C. Maass, C. Krüger, S. Herminghaus, and C. Bahr, Swimming droplets, Annu. Rev. Condens. Matter Phys. 7, 171 (2016).

[2] K. Peddireddy, P. Kumar, S. Thutupalli, S. Herminghaus, and C. Bahr, Solubilization of thermotropic liquid crystal compounds in aqueous surfactant solutions, Langmuir 28, 12426 (2012).

[3] S. Herminghaus, C. C. Maass, C. Krüger, S. Thutupalli, L. Goehring, and C. Bahr, Interfacial mechanisms in active emulsions, Soft Matter 10, 7008 (2014).

[4] C. Jin, C. Krüger, and C. C. Maass, Chemotaxis and autochemotaxis of self-propelling droplet swimmers, Proc. Natl. Acad. Sci. USA 114, 5089 (2017).
[5] C. Jin, B. V. Hokmabad, K. A. Baldwin, and C. C. Maass, Chemotactic droplet swimmers in complex geometries, J. Phys.: Condens. Matter 30, 054003 (2018).

[6] C. Krüger, C. Bahr, S. Herminghaus, and C. C. Maass, Dimensionality matters in the collective behaviour of active emulsions, Eur. Phys. J. E 39, 64 (2016).

[7] B. V. Hokmabad, R. Dey, M. Jalaal, D. Mohanty, M. Almukambetova, K. A. Baldwin, D. Lohse, and C. C. Maass, Emergence of Bimodal Motility in Active Droplets, Phys. Rev. X 11, 011043 (2021).

[8] C. Krüger, G. Klös, C. Bahr, and C. C. Maass, Curling Liquid Crystal Microswimmers: A Cascade of Spontaneous Symmetry Breaking, Phys. Rev. Lett. 117, 048003 (2016). 
[9] M. Suga, S. Suda, M. Ichikawa, and Y. Kimura, Self-propelled motion switching in nematic liquid crystal droplets in aqueous surfactant solutions, Phys. Rev. E 97, 062703 (2018).

[10] C. Krüger, Liquid crystal microswimmers-From single entities to collective dynamics, Ph.D. thesis, Faculty of Physics, Georg-August-Universität, Göttingen (2016).

[11] B. V. Hokmabad, K. A. Baldwin, C. Krüger, C. Bahr, and C. C. Maass, Topological Stabilization and Dynamics of SelfPropelling Nematic Shells, Phys. Rev. Lett. 123, 178003 (2019).

[12] M. Schmitt and H. Stark, Marangoni flow at droplet interfaces: Three-dimensional solution and applications, Phys. Fluids 28, 012106 (2016).

[13] A. Zöttl and H. Stark, Emergent behavior in active colloids, J. Phys.: Condens. Matter 28, 253001 (2016).

[14] L. Stricker, Numerical simulation of artificial microswimmers driven by Marangoni flow, J. Comput. Phys. 347, 467 (2017).

[15] M. Morozov and S. Michelin, Orientational instability and spontaneous rotation of active nematic droplets, Soft Matter 15, 7814 (2019).

[16] M. J. Lighthill, On the squirming motion of nearly spherical deformable bodies through liquids at very small Reynolds numbers, Commun. Pure Appl. Math. 5, 109 (1952).

[17] J. R. Blake, A spherical envelope approach to ciliary propulsion, J. Fluid Mech. 46, 199 (1971).

[18] S. Chen and G. D. Doolen, Lattice Boltzmann method for fluid flows, Annu. Rev. Fluid Mech. 30, 329 (1998).

[19] T. Krüger, H. Kusumaatmaja, A. Kuzmin, O. Shardt, G. Silva, and E. M. Viggen, The Lattice Boltzmann Method-Principles and Practice (Springer International Publishing, Cham, 2017).

[20] M. Bouzidi, M. Firdaouss, and P. Lallemand, Momentum transfer of a Boltzmann-lattice fluid with boundaries, Phys. Fluids 13, 3452 (2001).

[21] Z. Guo, C. Zheng, and B. Shi, Discrete lattice effects on the forcing term in the lattice Boltzmann method, Phys. Rev. E 65, 046308 (2002).

[22] A. J. C. Ladd and R. Verberg, Lattice-Boltzmann simulations of particle-fluid suspensions, J. Stat. Phys. 104, 1191 (2001).

[23] J. Happel and H. Brenner, Low Reynolds Number Hydrodynamics (Martinus Nijhoff Publishers, The Hague, 1983).

[24] T. Ishikawa and T. J. Pedley, Coherent Structures in Monolayers of Swimming Particles, Phys. Rev. Lett. 100, 088103 (2008).

[25] A. Sonnet, A. Kilian, and S. Hess, Alignment tensor versus director: Description of defects in nematic liquid crystals, Phys. Rev. E 52, 718 (1995).

[26] P. G. de Gennes, Short range order effects in the isotropic phase of nematics and cholesterics, Mol. Cryst. Liq. Cryst. 12, 193 (1971).

[27] C. Gähwiller, Temperature Dependence of Flow Alignment in Nematic Liquid Crystals, Phys. Rev. Lett. 28, 1554 (1972).

[28] C. J. Gerritsma, C. Z. van Doorn, and P. van Zanten, Transient effects in the electrically controlled light transmission of a twisted nematic layer, Phys. Lett. A 48, 263 (1974).

[29] M. Miesowicz, The three coefficients of viscosity of anisotropic liquids, Nature (London) 158, 27 (1946).

[30] J. L. Ericksen, Anisotropic fluids, Arch. Ration. Mech. Anal. 4, 231 (1960).

[31] F. M. Leslie, Some constitutive equations for anisotropic fluids, Q. J. Mech. Appl. Math. 19, 357 (1966).

[32] F. M. Leslie, Some constitutive equations for liquid crystals, Arch. Ration. Mech. Anal. 28, 265 (1968).
[33] B. J. Edwards, A. N. Beris, and M. Grmela, Generalized constitutive equation for polymeric liquid crystals. Part 1. Model formulation using the Hamiltonian (Poisson bracket) formulation, J. Non-Newt. Fluid Mech. 35, 51 (1990).

[34] A. N. Beris and B. J. Edwards, Thermodynamics of Flowing Systems with Internal Microstructure (Oxford University Press, Oxford, 1994).

[35] T. Qian and P. Sheng, Generalized hydrodynamic equations for nematic liquid crystals, Phys. Rev. E 58, 7475 (1998).

[36] C. M. Care, I. Halliday, and K. Good, Lattice Boltzmann nemato-dynamics, J. Phys.: Condens. Matter 12, L665 (2000).

[37] C. M. Care, I. Halliday, K. Good, and S. V. Lishchuk, Generalized lattice Boltzmann algorithm for the flow of a nematic liquid crystal with variable order parameter, Phys. Rev. E 67, 061703 (2003).

[38] C. Denniston, E. Orlandini, and J. M. Yeomans, Lattice Boltzmann simulations of liquid crystal hydrodynamics, Phys. Rev. E 63, 056702 (2001).

[39] C. Denniston, D. Marenduzzo, E. Orlandini, and J. M. Yeomans, Lattice Boltzmann algorithm for three-dimensional liquid-crystal hydrodynamics, Philos. Trans. R. Soc. London A 362, 1745 (2004).

[40] S. Mandal and M. G. Mazza, Multiparticle collision dynamics for tensorial nematodynamics, Phys. Rev. E 99, 063319 (2019).

[41] D. Marenduzzo, E. Orlandini, M. E. Cates, and J. M. Yeomans, Steady-state hydrodynamic instabilities of active liquid crystals: Hybrid lattice Boltzmann simulations, Phys. Rev. E 76, 031921 (2007).

[42] M. Ravnik and J. M. Yeomans, Confined Active Nematic Flow in Cylindrical Capillaries, Phys. Rev. Lett. 110, 026001 (2013).

[43] A. Sengupta, U. Tkalec, M. Ravnik, J. M. Yeomans, C. Bahr, and S. Herminghaus, Liquid Crystal Microfluidics for Tunable Flow Shaping, Phys. Rev. Lett. 110, 048303 (2013).

[44] V. M. O. Batista, M. L. Blow, and M. M. T. da Gama, The effect of anchoring on the nematic flow in channels, Soft Matter 11, 4674 (2015).

[45] R. Zhang, T. Roberts, I. S. Aranson, and J. J. de Pablo, Lattice Boltzmann simulation of asymmetric flow in nematic liquid crystals with finite anchoring, J. Chem. Phys. 144, 084905 (2016).

[46] J. S. Lintuvuori, D. Marenduzzo, K. Stratford, and M. E. Cates, Colloids in liquid crystals: A lattice Boltzmann study, J. Mater. Chem. 20, 10547 (2010).

[47] A. Antipova and C. Denniston, Dynamics of a disc in a nematic liquid crystal, Soft Matter 12, 1279 (2016).

[48] D. Marenduzzo, E. Orlandini, and J. M. Yeomans, Permeative Flows in Cholesteric Liquid Crystals, Phys. Rev. Lett. 92, 188301 (2004).

[49] O. Henrich, K. Stratford, P. V. Coveney, M. E. Cates, and D. Marenduzzo, Rheology of cubic blue phases, Soft Matter 9, 10243 (2013).

[50] J. M. Brake, A. D. Mezera, and N. L. Abbott, Effect of surfactant structure on the orientation of liquid crystals at aqueous-liquid crystal interfaces, Langmuir 19, 6436 (2003).

[51] A. Sengupta, S. Herminghaus, and C. Bahr, Opto-fluidic velocimetry using liquid crystal microfluidics, Appl. Phys. Lett. 101, 164101 (2012).

[52] See Supplemental Material at http://link.aps.org/supplemental/ 10.1103/PhysRevE.104.044703 for details of the mapping 
between physical units and LB units and for explicit expressions for the Jones matrices of optical elements and sample voxels.

[53] P. Oswald and P. Pieranski, Nematic and Cholesteric Liquid Crystals (Taylor \& Francis, Boca Raton, FL, 2005), Chap. III.4.

[54] O. D. Lavrentovich, Topological defects in dispersed liquid crystals, or words and worlds around liquid crystal drops, Liq. Cryst. 24, 117 (1998).

[55] T. Lopez-Leon and A. Fernandez-Nieves, Drops and shells of liquid crystal, Colloid Polym. Sci. 289, 345 (2011).

[56] M. Urbanski, C. G. Reyes, J. Noh, A. Sharma, Y. Geng, V. S. R. Jampani, and J. P. F. Lagerwall, Liquid crystals in micron-scale droplets, shells and fibers, J. Phys.: Condens. Matter 29, 133003 (2017).

[57] J. Yoshioka and K. Fukao, Horizontal transportation of a Maltese cross pattern in nematic liquid crystalline droplets under a temperature gradient, Phys. Rev. E 99, 022702 (2019).

[58] J. Yoshioka and K. Fukao, Horizontal transportation of a Maltese cross pattern in nematic liquid crystalline droplets under a direct-current electric field, J. Phys. Soc. Jpn. 89, 094401 (2020).

[59] A. Fernández-Nieves, D. R. Link, M. Márquez, and D. A. Weitz, Topological Changes in Bipolar Nematic Droplets under Flow, Phys. Rev. Lett. 98, 087801 (2007).

[60] M. Kleman and O. D. Lavrentovich, Topological point defects in nematic liquid crystals, Philos. Mag. 86, 4117 (2006).

[61] M. Ravnik and S. Zumer, Landau-de Gennes modelling of nematic liquid crystal colloids, Liq. Cryst. 36, 1201 (2009).

[62] H. Mori, E. C. Gartland, Jr., J. R. Kelly, and P. J. Bos, Multidimensional director modeling using the $\mathrm{Q}$ tensor representation in a liquid crystal cell and its application to the $\pi$ cell with patterned electrodes, Jpn. J. Appl. Phys., Part 1 38, 135 (1999).
[63] G.-D. Lee, P. J. Bos, S. H. Ahn, and K. H. Kim, Fast $Q$-tensor method for modeling the dynamics of defects in a liquid crystal director field, Phys. Rev. E 67, 041715 (2003).

[64] R. C. Jones, A new calculus for the treatment of optical systems, J. Opt. Soc. Am. 31, 488 (1941).

[65] R. M. A. Azzam and N. M. Bashara, Ellipsometry and Polarized Light (Elsevier Science, Amsterdam, 1987), p. 99.

[66] R. Ondris-Crawford, E. P. Boyko, B. G. Wagner, J. H. Erdmann, S. Zumer, and J. W. Doane, Microscope textures of nematic droplets in polymer dispersed liquid crystals, J. Appl. Phys. 69, 6380 (1991).

[67] J. Ding and Y. Yang, Birefringence patterns of nematic droplets, Jpn. J. Appl. Phys. 31, 2837 (1992).

[68] J. Jeong, Z. S. Davidson, P. J. Collings, T. C. Lubensky, and A. G. Yodha, Chiral symmetry breaking and surface faceting in chromonic liquid crystal droplets with giant elastic anisotropy, Proc. Natl. Acad. Sci. USA 111, 1742 (2014).

[69] U. Mur, S. Copar, G. Posnjak, I. Musevic, M. Ravnik, and S. Zumer, Ray optics simulations of polarised microscopy textures in chiral nematic droplets, Liq. Cryst. 44, 679 (2017).

[70] S.-T. Wu, C.-S. Wu, M. Warenghem, and M. Ismaili, Refractive index dispersions of liquid crystals, Opt. Engin. 32, 1775 (1993).

[71] T. Smith and J. Guild, The C.I.E. colorimetric standards and their use, Trans. Opt. Soc. 33, 73 (1931).

[72] X. Shan and H. Chen, Lattice Boltzmann model for simulating flows with multiple phases and components, Phys. Rev. E 47, 1815 (1993).

[73] M. R. Swift, E. Orlandini, W. R. Osborn, and J. M. Yeomans, Lattice Boltzmann simulations of liquid-gas and binary fluid systems, Phys. Rev. E 54, 5041 (1996). 